CIRJE-F-796

\title{
Rebalancing Static Super-Replications
}

\author{
Akihiko Takahashi \\ University of Tokyo \\ Yukihiro Tsuzuki \\ Mizuho-DL Financial Technology Co., Ltd.
}

April 2011; Revised in July 2011

CIRJE Discussion Papers can be downloaded without charge from:

http://www.cirje.e.u-tokyo.ac.jp/research/03research02dp.html

Discussion Papers are a series of manuscripts in their draft form. They are not intended for circulation or distribution except as indicated by the author. For that reason Discussion Papers may not be reproduced or distributed without the written consent of the author. 


\title{
Rebalancing Static Super-Replications *
}

\author{
Akihiko Takahashi † Yukihiro Tsuzuki ${ }^{\ddagger}$
}

July 26, 2011

\begin{abstract}
This paper proposes a trading strategy that dynamically rebalances static super-replicating portfolios, which is very useful for both investment and hedging strategies. In order to investigate general properties of the strategy, we derive the Doob-Meyer decomposition for the value process without any specifications of models under the continuous processes of the underlying variables. In particular, we find that the increasing part of the decomposition characterizes the performance of the strategy. Also, we obtain more concrete features for cross-currency and one-touch options based on our general framework. Moreover, numerical examples for cross-currency options demonstrate the effectiveness of our strategy for investment and hedging.
\end{abstract}

\footnotetext{
*All the contents expressed in this research are solely those of the authors and do not represent the view of Mizuho-DL Financial Technology Co.,Ltd. or any other institutions. The authors are not responsible or liable in any manner for any losses and/or damages caused by the use of any contents in this research.

${ }^{\dagger}$ Graduate School of Economics, University of Tokyo, 7-3-1, Hongo, Bunkyo-ku, Tokyo, 113-0033, Japan.

${ }^{\ddagger}$ Mizuho-DL Financial Technology Co., Ltd., 1-3, Otemachi 1-chome, Chiyoda-ku, Tokyo 100-0004, Japan. E-mail: yukihiro-tsuzuki@fintec.co.jp
} 


\section{Introduction}

This paper introduces a trading strategy that dynamically rebalances static super-replicating portfolios, which is very attractive for both investment and hedging. Specifically, we derive the Doob-Meyer decomposition for the value process of this strategy without any specifications of models under the continuous processes of the underlying variables: the increasing part of the decomposition is a key element since it characterizes the performance of the strategy.

Super-replications have been more attractive since 2007 after the financial crisis, because they provide a protection against substantial losses. In particular, thanks to the robustness of their model-independent properties, they put a rigid floor on the maximum loss whatever the subsequent paths of the underlying prices.

The problem of finding the cheapest super-replication has first been introduced by El Karoui and Quenez [4] for the case of dynamic trading strategies. Subsequently, various types of super-replicating strategies have been proposed. Among them, several model-independent and static/semistatic super-replications have been investigated by Chung and Wang [2], Neuberger and Hodges [6] and Tsuzuki [7]. Here, static replication is a method of replicating a derivative with portfolio whose composition does not change until the maturity of the derivative and semi-static one is a method of replication by trading no more than once after inception. While they are robust, these strategies have a serious drawback that the probability of suffering the maximum loss is extremely high.

In order to overcome this drawback, we propose a dynamically rebalancing strategy of the cheapest super-replication. As an intuitive explanation for the feature of this strategy, consider static super-replications whose portfolios are derived as the cheapest among some family of superreplicating portfolios. First, the super-replicating portfolio is constructed as the cheapest one. After the market conditions have changed, the original portfolio is no longer the cheapest and another one becomes the cheapest. By liquidating the original one and constructing the new cheapest one, an amount of cash is withdrawn from the position:this amount should be positive because the latter is cheaper than the former. The strategy continues this operation until the maturity. Then, thanks to the accumulation of these positive cash flows, the probability of the maximum loss is reduced. We remark that Neuberger and Hodges [6] examines a numerical example of this type of strategy for barrier options. (Dupire [3] called it "roll-down".)

This paper analyzes properties of the strategy without assuming any models under the continuous processes of the underlying variables. In particular, to the best of our knowledge, it is the first work that derives the Doob-Meyer decomposition for the value process, which is a super-martingale process because it is defined as an infimum of a certain family of portfolios. Moreover, we give a financial interpretation to the decomposition and obtain general properties of the strategy through the increasing part of the decomposition;the increasing part is practically important because it characterizes the performance of the strategy. More concrete features become known by applications of our results to specific 
derivatives such as cross-currency and one-touch options under some additional assumptions that are satisfied for usual cases. Further, numerical examples for cross-currency options demonstrate the effectiveness of our strategy for both investment and hedging.

The organization of this paper is as follows: Section 2 states assumptions and notations. Section 3 is devoted to our main theorem on the Doob-Meyer decomposition. Section 4 applies our result to cross-currency and one-touch options. Numerical examples for cross-currency options are demonstrated in Section 5. The last section gives concluding remarks. Appendix presents analytical results for cross-currency options under the Black-Scholes model.

\section{Assumptions and Notations}

We consider the problem of hedging a derivative by liquid instruments such as bonds, risky assets as well as plain-vanilla options on those assets in a frictionless and no-arbitrage market, which is defined on a filtered probability space $\left(\Omega, \mathcal{F},\left\{\mathcal{F}_{t}\right\}_{t \in\left[0, T^{*}\right]}, \mathbb{Q}\right)$ for some arbitrary time horizon $T^{*}>0$. The no-arbitrage condition ensures the existence of a risk-neutral measure $\mathbb{Q}$ such that the instantaneous expected rate of return on every asset is equal to the instantaneous interest rate. For sake of simplicity, the interest rate and the dividend yields are assumed to be zero.

Let $\mathcal{O}_{X}$ be a domain of $\mathbb{R}^{N}$ and $X: \Omega \times\left[0, T^{*}\right] \longrightarrow \mathcal{O}_{X}$ be an $N$ dimensional $\left\{\mathcal{F}_{t}\right\}$-adapted continuous process which represents all the underlying random variables such as asset prices and their volatilities:

$$
\begin{aligned}
& X_{t}:=X_{0}+A_{t}+M_{t} \\
& X_{0}:=x,
\end{aligned}
$$

where $A_{t}$ is an $N$-dimensional finite variation process, $M_{t}$ is an $N$-dimensional continuous local martingale and $x \in \mathcal{O}_{X}$. The $i$-th component of the each vector is expressed by $X_{t}^{(i)}, A_{t}^{(i)}$ or $M_{t}^{(i)}$.

Let $Y: \Omega \times\left[0, T^{*}\right] \longrightarrow \mathbb{R}^{D}$ be an $D$-dimensional process which denotes time- $t$ prices of all tradable securities, which is $\left\{\mathcal{F}_{t}\right\}$-adapted and continuous. Note that $Y$ is a local martingale under our assumption that the interest rate is zero. We define a trading strategy $\phi$ by an $D$-dimensional $\left\{\mathcal{F}_{t}\right\}$-adapted process $\phi: \Omega \times[0, T] \longrightarrow \mathbb{R}^{D}$, whose $i$-th component is the number of the security $Y^{(i)}$ held by the strategy, where $T$ is the end of a trading period. In particular, a trading strategy $\phi$ is a semi-static strategy if and only if $\phi$ is constant on $[0, \tau)$ and $(\tau, T]$ for some stopping time $\tau$ and a strategy is static if and only if $\tau=T$.

Let $Y^{*}: \Omega \times\left[0, T^{*}\right] \longrightarrow \mathbb{R}$ be a price process of the derivative to be hedged, which is assumed to be $\left\{\mathcal{F}_{t}\right\}$-adapted. $Y_{t}^{*}$ may not be measurable with respect to the $\sigma$-algebra generated by $Y_{t}$ for any $t \in\left[0, T^{*}\right]$, which means that the derivative $Y^{*}$ can not be replicated by the tradable securities. Then, we consider the strategy that super-replicates the derivative. Here, a super-replicating strategy of $Y_{t}^{*}$ is a trading strategy such that 
for any $t \in\left[0, T^{*}\right]$,

$$
Y_{t}^{*} \leq \sum_{n=1}^{D} \phi_{t}^{(n)} Y_{t}^{(n)} .
$$

In particular, suppose that $\phi$ is a semi-static super-replicating strategy for a stopping time $\tau$. Then, for $t \in[0, \tau)$

$$
Y_{t}^{*} \leq \sum_{n=1}^{D} \phi_{0}^{(n)} Y_{t}^{(n)} .
$$

Assume that there exists a family of static strategies that super-replicate the derivative $Y^{*}$. Let us denote the time- $t$ prices of static portfolios in the family by $\{H(t, x, K)\}_{K \in \mathcal{O}_{K}}$, where $H$ is assumed to depend on a parameter $K \in \mathcal{O}_{K}$ with some domain $\mathcal{O}_{K}$ in $\mathbb{R}$ as well as on the time parameter $t$ and market variables $x \in \mathcal{O}_{X}$.

Remark 1. Some derivatives can be super-replicated by portfolios consisting of plain-vanilla options whose strike prices are arbitrary. In these cases, the parameter $K$ of $\{H(t, x, K)\}_{K \in \mathcal{O}_{K}}$ corresponds to the strike price. We will look at those examples in Section 4.

\section{Rebalancing Super-Replications}

This section investigates the strategy which dynamically rebalances superreplications.

The strategy is explained as follows. First, the super-replicating portfolio is constructed at time $t=0$ as the cheapest one by solving the optimization problem (3.1) below. Then, rebalancing the super-replicating portfolio is continuously executed until the maturity $T$. This is carried out by solving the optimization problem (3.1) under the market conditions at time $t$. It is noteworthy that the strategy is not self-financed because an amount of cash is extracted from the position until the maturity $T$. The performance of the strategy depends on how much these cash flows are. In order to investigate them, we derive the Doob-Meyer decomposition of the process $\left\{H_{t}^{*}\right\}_{t \in[0, T]}$, which is the value process of the strategy.

Some assumptions and lemmas are necessary for obtaining the decomposition.

Assumption 1. $H(t, x, K)$ is assumed to have the unique infimum value with respect to $K$ for all $t \in[0, T)$ and $x \in \mathcal{O}_{X}$. Let $H^{*}(t, x)$ be the infimum value and $K^{*}(t, x)$ be a point where the infimum is attained:

$$
\begin{aligned}
H^{*}(t, x) & :=\inf _{K>0} H(t, x, K) \\
& =H\left(t, x, K^{*}(t, x)\right) .
\end{aligned}
$$

Hereafter, the following notations will be used for simplicity: $H_{t}(K):=$ $H\left(t, X_{t}, K\right), K_{t}^{*}:=K^{*}\left(t, X_{t}\right)$ and so on.

Assumption 2. $H(t, x, \cdot)$ and $K^{*}(t, x)$ are sufficiently smooth with respect to $t$ and $x$. 
The process $H_{t}(K)$ for each $K \in \mathcal{O}_{K}$ is the price process of the static position of a super-replicating portfolio. Under the assumption that the interest rate is assumed to be zero, the process is a local martingale.

Lemma 1. Suppose $t>0$ and $K \in \mathcal{O}_{K}$. Then

$$
\begin{aligned}
& \int_{0}^{t} \frac{\partial H}{\partial t}\left(s, X_{s}, K\right) d s+\sum_{i=1}^{N} \int_{0}^{t} \frac{\partial H}{\partial x_{i}}\left(s, X_{s}, K\right) d A_{s}^{(i)} \\
& +\sum_{i, j<N} \int_{0}^{t} \frac{1}{2} \frac{\partial^{2} H}{\partial x_{i} \partial x_{j}}\left(s, X_{s}, K\right) d\left\langle M^{(i)}, M^{(j)}\right\rangle_{s}=0
\end{aligned}
$$

and

$$
H_{t}(K)=H_{0}(K)+\sum_{i=1}^{N} \int_{0}^{t} \frac{\partial H}{\partial x_{i}}\left(s, X_{s}, K\right) d M_{s}^{(i)} .
$$

Proof. By Ito's formula, we have for $\forall t>s>0, \forall K \in \mathcal{O}_{K}$

$$
\begin{aligned}
H_{t}(K)= & H_{s}(K)+\int_{s}^{t} \frac{\partial H}{\partial t}\left(u, X_{u}, K\right) d u \\
& +\sum_{i=1}^{N} \int_{s}^{t} \frac{\partial H}{\partial x_{i}}\left(u, X_{u}, K\right) d X_{u}^{(i)} \\
& +\sum_{i, j<N} \int_{s}^{t} \frac{1}{2} \frac{\partial^{2} H}{\partial x_{i} \partial x_{j}}\left(u, X_{u}, K\right) d\left\langle M^{(i)}, M^{(j)}\right\rangle_{u} .
\end{aligned}
$$

Since the process $\left\{H_{t}(K)\right\}_{t \in[0, T]}$ is a local martingale, Eq.(3.2) and Eq.(3.3) are obtained.

Theorem 1 depends on the following assumption, which is satisfied in usual cases where the optimization problem (3.1) admits the unique solution.

Assumption 3. $H(t, x, K)$ is twice continuously differentiable in the neighborhood of $K^{*}(t, x)$ with respect to $K$ for all $t \in[0, T)$ and $x \in \mathcal{O}_{X}$. Moreover,

$$
\begin{aligned}
\frac{\partial H}{\partial K}\left(t, x, K^{*}(t, x)\right) & =0 \\
\frac{\partial^{2} H}{\partial K^{2}}\left(t, x, K^{*}(t, x)\right) & >0 .
\end{aligned}
$$

Theorem 1. Suppose Assumption 3. Then, the process $\left\{H_{t}^{*}\right\}_{t \in[0, T]}$ is a super-martingale and its Doob-Meyer decomposition is given by

$$
H_{t}^{*}=H_{0}^{*}-A_{t}^{*}+M_{t}^{*}
$$

where the process $\left\{A_{t}^{*}\right\}_{t \in[0, T]}$ is increasing:

$$
A_{t}^{*}=\int_{0}^{t} \frac{\partial^{2} H}{\partial K^{2}}\left(s, X_{s}, K_{s}^{*}\right) d\left\langle K^{*}\right\rangle_{s}
$$


and the process $\left\{M_{t}^{*}\right\}_{t \in[0, T]}$ is a martingale:

$$
M_{t}^{*}=\sum_{i=1}^{N} \int_{0}^{t} \frac{\partial H}{\partial x_{i}}\left(s, X_{s}, K_{s}^{*}\right) d M_{s}^{(i)} .
$$

Proof. By Assumption 3, we have for any $t \in[0, T], x \in \mathcal{O}_{X}$,

$$
\begin{aligned}
& \frac{\partial H}{\partial K}\left(t, x, K^{*}(t, x)\right) \frac{\partial K^{*}}{\partial t}(t, x)=0 \\
& \frac{\partial H}{\partial K}\left(t, x, K^{*}(t, x)\right) \frac{\partial K^{*}}{\partial x_{i}}(t, x)=0
\end{aligned}
$$

and

$$
\begin{aligned}
& \frac{\partial}{\partial x_{i}}\left(\frac{\partial H}{\partial K}\left(t, x, K^{*}(t, x)\right)\right) \\
= & \frac{\partial^{2} H}{\partial x_{i} \partial K}\left(t, x, K^{*}(t, x)\right)+\frac{\partial^{2} H}{\partial K^{2}}\left(t, x, K^{*}(t, x)\right) \frac{\partial K^{*}}{\partial x_{i}}(t, x) \\
= & 0 .
\end{aligned}
$$

Ito's formula implies that

$$
\begin{aligned}
H_{t}^{*}= & H_{0}^{*}+\int_{0}^{t} \frac{\partial H^{*}}{\partial t}\left(s, X_{s}\right) d s \\
& +\sum_{i=1}^{N} \int_{0}^{t} \frac{\partial H^{*}}{\partial x_{i}}\left(s, X_{s}\right) d X_{s}^{(i)}+\sum_{i, j<N} \int_{0}^{t} \frac{1}{2} \frac{\partial^{2} H^{*}}{\partial x_{i} \partial x_{j}}\left(s, X_{s}\right) d\left\langle X^{(i)}, X^{(j)}\right\rangle_{s} \\
= & H_{0}^{*}+\int_{0}^{t}\left(\frac{\partial H}{\partial t}\left(s, X_{s}, K_{s}^{*}\right)+\frac{\partial H}{\partial K}\left(s, X_{s}, K_{s}^{*}\right) \frac{\partial K^{*}}{\partial t}\left(s, X_{s}\right)\right) d s \\
& +\sum_{i=1}^{N} \int_{0}^{t}\left(\frac{\partial H}{\partial x_{i}}\left(s, X_{s}, K_{s}^{*}\right)+\frac{\partial H}{\partial K}\left(s, X_{s}, K_{s}^{*}\right) \frac{\partial K^{*}}{\partial x_{i}}\left(s, X_{s}\right)\right) d X_{s}^{(i)} \\
& +\sum_{i, j<N} \int_{0}^{t} \frac{1}{2}\left(\frac{\partial^{2} H}{\partial x_{i} \partial x_{j}}\left(\cdot, K_{s}^{*}\right)+\frac{\partial^{2} H}{\partial x_{i} \partial K}\left(\cdot, K_{s}^{*}\right) \frac{\partial K^{*}}{\partial x_{j}}(\cdot)\right)\left(s, X_{s}\right) d\left\langle X^{(i)}, X^{(j)}\right\rangle_{s} \\
= & H_{0}^{*}+\sum_{i=1}^{N} \int_{0}^{t} \frac{\partial H}{\partial x_{i}}\left(s, X_{s}, K_{s}^{*}\right) d M_{s}^{(i)} \\
& +\sum_{i, j<N} \int_{0}^{t} \frac{1}{2} \frac{\partial^{2} H}{\partial x_{i} \partial K}\left(s, X_{s}, K_{s}^{*}\right) \frac{\partial K^{*}}{\partial x_{j}}\left(s, X_{s}\right) d\left\langle X^{(i)}, X^{(j)}\right\rangle_{s},
\end{aligned}
$$

where we have used Lemma 1.

Let the first integral be defined as $M_{t}^{*}$ and the last one be defined as $-A_{t}^{*}$. Then, we have

$$
\begin{aligned}
A_{t}^{*} & =-\sum_{i, j<N} \int_{0}^{t} \frac{1}{2} \frac{\partial^{2} H}{\partial x_{i} \partial K}\left(s, X_{s}, K_{s}^{*}\right) \frac{\partial K^{*}}{\partial x_{j}}\left(s, X_{s}\right) d\left\langle X^{(i)}, X^{(j)}\right\rangle_{s} \\
& =\sum_{i, j<N} \frac{1}{2} \int_{0}^{t} \frac{\partial^{2} H}{\partial K^{2}}\left(s, X_{s}, K_{s}^{*}\right) \frac{\partial K^{*}}{\partial x_{i}}\left(s, X_{s}\right) \frac{\partial K^{*}}{\partial x_{j}}\left(s, X_{s}\right) d\left\langle X^{(i)}, X^{(j)}\right\rangle_{s} \\
& =\frac{1}{2} \int_{0}^{t} \frac{\partial^{2} H}{\partial K^{2}}\left(s, X_{s}, K_{s}^{*}\right) d\left\langle\sum_{i=1}^{N} \int_{0}^{\cdot} \frac{\partial K^{*}}{\partial x_{i}} d X^{(i)}\right\rangle_{s}
\end{aligned}
$$


It is found that the process $\left\{M_{t}^{*}\right\}_{t \in[0, T]}$ is a martingale and $\left\{A_{t}^{*}\right\}_{t \in[0, T]}$ is increasing by Assumption 3 .

Remark 2. In case where $H^{*}(t, x)$ takes the infimum value at a boundary point, Eq.(3.5) of Assumption 3 may not hold. Nevertheless, Theorem 1 holds under an assumption that $K^{*}(t, x)$ is constant on the subset of $[0, T) \times \mathcal{O}_{X}$ that $H^{*}(t, x)$ takes the infimum value at a boundary point, because Eq.(3.10) and (3.11) hold and $A_{t}^{*}=0$ on the set.

A financial interpretation of Theorem 1 is as follows. The variation of the martingale part $M$ is approximated by the difference between the time$t$ value and the time-s value of the time- $s$ optimal portfolio by Eq.(3.3) for all $s<t$, which is a variation of the price of a portfolio held at time $s$ :

$$
\begin{aligned}
M_{t}^{*}-M_{s}^{*} & =\sum_{i=1}^{N} \int_{s}^{t} \frac{\partial H}{\partial x_{i}}\left(u, X_{u}, K_{u}^{*}\right) d M_{u}^{(i)} \\
& \approx \sum_{i=1}^{N} \frac{\partial H}{\partial x_{i}}\left(s, X_{s}, K_{s}^{*}\right) \Delta M_{s}^{(i)} \\
& \approx H_{t}\left(K_{s}^{*}\right)-H_{s}\left(K_{s}^{*}\right),
\end{aligned}
$$

where $\Delta M_{s}^{(i)}:=M_{t}^{(i)}-M_{s}^{(i)}$ and we have used the approximation

$$
H_{t}(K)-H_{s}(K) \approx \sum_{i=1}^{N} \frac{\partial H}{\partial x_{i}}\left(s, X_{s}, K\right) \Delta M_{s}^{(i)}
$$

with $K=K_{s}^{*}$.

Then, we obtain

$$
A_{t}^{*}-A_{s}^{*} \approx H_{t}\left(K_{t}^{*}\right)-H_{t}\left(K_{s}^{*}\right),
$$

which is the difference between the time- $t$ value of the time- $t$ optimal portfolio and that of the time- $s$ optimal portfolio. Hence, the increasing part $A$ is regarded as the accumulation of cash flows generated by each rebalancing. Consequently, the value process for a trader shorting the derivative with the optimally rebalancing super-replication strategy is given by

$$
H_{t}^{*}+A_{t}^{*}-Y_{t}^{*}=H_{0}^{*}+M_{t}^{*}-Y_{t}^{*}
$$

This implies that the larger is $A_{t}^{*}$, the more profitable the strategy is.

A general property is derived through this analysis. Eq.(3.8) shows that the time- $t$ cash flow is a change in the quadratic variation of the optimal parameter $K^{*}$ weighted by the second-order derivative of $H$ with respect to the parameter $K$ at $K=K^{*}$. The former represents the extent of the fluctuation of the optimal parameter $K^{*}$ while the latter expresses the extent of convexity of the function $H(t, x, \cdot)$ at an optimal point $K^{*}$. For further detailed properties, we study some specific options in the next section. 


\section{Applications}

This section applies our result to cross-currency and one-touch options, where we assume conditions normally satisfied for plain-vanilla European options;for instance, the prices of these options are sufficiently smooth with respect to every parameter and the delta and the vega ${ }^{1}$ of call options are positive.

\subsection{Cross-Currency Options}

This subsection applies Theorem 1 to cross-currency European options. Let a currency exchange rate $X_{T}^{(i)}$ be the price of the unit amount of Currency $i$ in terms of a base currency such as USD(U.S. dollar). Consider a cross-currency rate representing the price of the unit amount of Currency 1 in terms of Currency 2. Then, the payoff of a call option on the crosscurrency with strike 1 and maturity $T$ (in terms of Currency 2) is given by

$$
\left(X_{T}^{(1)} / X_{T}^{(2)}-1\right)_{+}=\left(1 / X_{T}^{(2)}\right)\left(X_{T}^{(1)}-X_{T}^{(2)}\right)_{+} .
$$

Hence, for pricing this option we need to evaluate an exchange option(see Margrabe [5]) whose payoff is $\left(X_{T}^{(1)}-X_{T}^{(2)}\right)_{+}$.

Next, note that the following super-/sub-replication is a well-known strategy.

Lemma 2. For all $K>0$, the payoff of an exchange option with maturity $T$ must satisfy the following inequalities:

$$
\begin{aligned}
\left(X_{T}^{(1)}-X_{T}^{(2)}\right)_{+} \leq & \left(X_{T}^{(1)}-K\right)_{+}+\left(K-X_{T}^{(2)}\right)_{+} \\
\left(X_{T}^{(1)}-X_{T}^{(2)}\right)_{+} \geq & \max \left\{\left(X_{T}^{(1)}-K\right)_{+}-\left(X_{T}^{(2)}-K\right)_{+},\right. \\
& \left.-\left(K-X_{T}^{(1)}\right)_{+}+\left(K-X_{T}^{(2)}\right)_{+}\right\} .
\end{aligned}
$$

Proof. Suppose $z_{1}, z_{2}, k \in \mathbb{R}$. Then

$$
\begin{aligned}
\left(z_{1}-z_{2}\right)_{+} & =\left(\left(z_{1}-k\right)+\left(k-z_{2}\right)\right)_{+} \\
& \leq\left(z_{1}-k\right)_{+}+\left(k-z_{2}\right)_{+} .
\end{aligned}
$$

by Jensen's inequality. If we substitute $z_{1}=X_{T}^{(1)}, z_{2}=X_{T}^{(2)}, k=K$ in Inequality (4.3), then Inequality (4.1) is derived. If we substitute $z_{1}=$ $X_{T}^{(1)}, z_{2}=K, k=X_{T}^{(2)}$ and $z_{1}=K, z_{2}=X_{T}^{(2)}, k=X_{T}^{(1)}$ in Inequality (4.3), then Inequality (4.2) is derived.

Let $C^{(i)}(t, x, K)$ and $P^{(i)}(t, x, K)$ be the time- $t$ prices of call and put options on the exchange rate $i(i=1,2)$ respectively, where $x \in \mathcal{O}_{X}$ is a $N$-dimensional vector consisting of all parameters relevant for option prices, such as the underlying exchange rates and their volatilities. Especially, we assume that the first component $x_{1}$ and the second one $x_{2}$ stand for the underlying exchange rates. The value process of the super/sub-replicating portfolio which corresponds to $H$ in the previous section is given as follows:

\footnotetext{
${ }^{1}$ The delta(vega) is the derivative with respect to the price(volatility) of the underlying asset price.
} 
Definition 1. Let $G$ and $L$ be the value of super-/sub-replicating portfolios with the strike $K$ :

$$
\begin{aligned}
G(t, x, K) & :=C^{(1)}(t, x, K)+P^{(2)}(t, x, K) \\
L(t, x, K) & :=-\max \left\{L^{C}(t, x, K), L^{P}(t, x, K)\right\},
\end{aligned}
$$

where

$$
\begin{aligned}
& L^{C}(t, x, K):=C^{(1)}(t, x, K)-C^{(2)}(t, x, K), \\
& L^{P}(t, x, K):=-P^{(1)}(t, x, K)+P^{(2)}(t, x, K) .
\end{aligned}
$$

In addition, let $K^{G}$ and $K^{L}$ be the optimal strike prices for super-/subreplication respectively.

Remark 3. The optimal strike prices $K^{G}$ and $K^{L}$ become the same as those in Chung and Wang [2].

We see if Assumption 3 is satisfied for these strategies. The optimal super-replicating portfolio is always determined uniquely as in the following lemma. On the other hand, the optimal sub-replicating portfolio is not always determined uniquely. We show a sufficient condition for uniqueness under the Black-Scholes model(see Appendix A).

Lemma 3. Suppose $H=G$. Then, Assumptions 1 and 3 hold.

Proof. By differentiating $G(t, x, K)$ with respect to $K$,

$$
\begin{aligned}
\frac{\partial G}{\partial K}(t, x, K) & =\frac{\partial C^{(1)}}{\partial K}(t, x, K)+\frac{\partial P^{(2)}}{\partial K}(t, x, K) \\
& =\frac{\partial C^{(1)}}{\partial K}(t, x, K)+\frac{\partial C^{(2)}}{\partial K}(t, x, K)+1 \\
\frac{\partial^{2} G}{\partial K^{2}}(t, x, K) & =\frac{\partial^{2} C^{(1)}}{\partial K^{2}}(t, x, K)+\frac{\partial^{2} C^{(2)}}{\partial K^{2}}(t, x, K) .
\end{aligned}
$$

The fact that $\frac{\partial^{2} C^{(i)}}{\partial K^{2}}(t, x, K)>0$ implies $\frac{\partial^{2} G}{\partial K^{2}}(t, x, K)>0$. Since $\frac{\partial G}{\partial K}(t, x, 0)=$ -1 and $\frac{\partial G}{\partial K}(t, x,+\infty)=+1, G(t, x, K)$ has the infimum value at $K$ which satisfies $\frac{\partial G}{\partial K}(t, x, K)=0$.

By investigating the property of $K^{*}$, it is found that $K^{*}$ depends on the correlation of the two underlying exchange rates.

Proposition 1. Suppose Assumption 3 holds for sub-replication. Then $\frac{\partial K_{s}^{G}}{\partial x_{1}} \frac{\partial K_{s}^{G}}{\partial x_{2}}>0$ and $\frac{\partial K_{s}^{L}}{\partial x_{1}} \frac{\partial K_{s}^{L}}{\partial x_{2}}<0$.

Proof. By Assumption 3,

$$
\frac{\partial P^{(1)}}{\partial K}\left(t, x, K^{G}(t, x)\right)+\frac{\partial P^{(2)}}{\partial K}\left(t, x, K^{G}(t, x)\right)-1=0
$$

and

$$
\frac{\partial P^{(1)}}{\partial K}\left(t, x, K^{L}(t, x)\right)=\frac{\partial P^{(2)}}{\partial K}\left(t, x, K^{L}(t, x)\right) .
$$


By differentiating these equations with respect to $x_{i}$ for $i=1,2$, we obtain

$$
\frac{\partial^{2} G}{\partial K^{2}}\left(t, x, K^{G}(t, x)\right) \frac{\partial K^{G}}{\partial x_{i}}=-\frac{\partial^{2} P^{(i)}}{\partial K \partial x_{i}}\left(t, x, K^{G}(t, x)\right)
$$

and

$$
\frac{\partial^{2} \tilde{L}}{\partial K^{2}}\left(t, x, K^{L}(t, x)\right) \frac{\partial K^{L}}{\partial x_{i}}=(-1)^{i+1} \frac{\partial^{2} P^{(i)}}{\partial K \partial x_{i}}\left(t, x, K^{L}(t, x)\right),
$$

where $\tilde{L}=-L^{C}$ or $L^{P} \cdot \frac{\partial^{2} P^{(i)}}{\partial K \partial x_{i}}$ is negative because the probability that the price at the maturity is less than any value goes down if a spot price goes up, and vice versa. This leads to the proposition.

Remark 4. Proposition 1 shows that the integrand $\frac{\partial K_{s}^{*}}{\partial x_{1}} \frac{\partial K_{s}^{*}}{\partial x_{2}}$ of the last term in the following approximation is positive for $K^{*}=K^{G}$ and negative for $K^{*}=K^{L}$ :

$$
\begin{aligned}
\left\langle K^{*}\right\rangle_{t} \approx & \int_{0}^{t}\left(\frac{\partial K_{s}^{*}}{\partial x_{1}}\right)^{2} d\left\langle X^{(1)}\right\rangle_{s}+\int_{0}^{t}\left(\frac{\partial K_{s}^{*}}{\partial x_{2}}\right)^{2} d\left\langle X^{(2)}\right\rangle_{s} \\
& +\int_{0}^{t} \frac{\partial K_{s}^{*}}{\partial x_{1}} \frac{\partial K_{s}^{*}}{\partial x_{2}} d\left\langle X^{(1)}, X^{(2)}\right\rangle_{s} .
\end{aligned}
$$

This implies the following relations approximately hold:

(i) The quadratic variation of $K^{G}$ is positively correlated to the quadratic covariation of $X^{(1)}$ and $X^{(2)}$.

(ii) The quadratic variation of $K^{L}$ is negatively correlated to the quadratic covariation of $X^{(1)}$ and $X^{(2)}$.

Note that our strategy is useful for an investment in the correlation of the two exchange rates. (See Section 5.1.)

\subsection{One-Touch Options}

We apply Theorem 1 to one-touch options. A one-touch option with maturity $T$ and a barrier level $B \in \mathbb{R}_{+}$is an option which is worthless if the barrier has not been hit, and pays one at the maturity if the barrier has been hit. Let the event that the barrier has been hit be

$$
A:=\left\{\omega \in \Omega \mid S_{t} \notin I \text { for some } t \in[0, T)\right\},
$$

where $I:=[0, B]$ and $S_{t}$ is the time- $t$ price of the underlying asset with $S_{0}<B$. Then, the payoff is $1_{A}$.

Brown et al. [1] and Neuberger and Hodges [6] introduced a modelindependent static super-replication under the assumption that the riskfree interest rate and the dividend yield of the underlying asset are zero and that the underlying asset process is continuous ${ }^{2}$. They consider the following family of strategies parameterized by a strike price $K \in[0, B)$ for a short position of a one-touch option. The strategy consists of at most two steps: the first step is to buy $(B-K)^{-1}$ amounts of a European

${ }^{2}$ If the assumption fails, their results are weakened. 
call option with strike $K$ at the beginning; the second step is to sell the call option and buy $(B-K)^{-1}$ amounts of a European put option with strike $K$ at the first hitting time. By the put-call parity, one amount of cash is left after the operation at the first hitting time.

We apply our theorem to this strategy until the first hitting time. Then, the value of the super-replicating portfolio is given by

$$
H(t, x, K)=\frac{C(t, x, K)}{B-K},
$$

where $C(t, x, K)$ is the time- $t$ price of a European call option and $x \in \mathcal{O}_{X}$ is a $N$-dimensional vector consisting of all parameters relevant for option prices such as the underlying price $S$ and its volatility $\sigma$.

Lemma 4. $H(t, x, K)$ satisfies Assumption 1 and 3.

Proof. By differentiating with respect to $K$, we obtain

$$
\frac{\partial H}{\partial K}(t, x, K)=\frac{g(t, x, K)}{(B-K)^{2}}
$$

and

$$
\frac{\partial^{2} H}{\partial K^{2}}(t, x, K)=\frac{1}{B-K} \frac{\partial^{2} C}{\partial K^{2}}(t, x, K)+\frac{2 g(t, x, K)}{(B-K)^{3}},
$$

where

$$
g(t, x, K):=\frac{\partial C}{\partial K}(t, x, K)(B-K)+C(t, x, K) .
$$

$\frac{\partial H}{\partial K}=0$ has solutions by the fact that $\frac{\partial H}{\partial K}(t, x, 0)=\frac{1}{B}\left(-1+\frac{S_{t}}{B}\right)<0$ and $\lim _{K \rightarrow B} \frac{\partial H}{\partial K}(t, x, K)=+\infty$. At these points, we have

$$
\frac{\partial^{2} H}{\partial K^{2}}(t, x, K)=\frac{1}{B-K} \frac{\partial^{2} C}{\partial K^{2}}(t, x, K)>0,
$$

which leads to the uniqueness of the solution.

Let us investigate the properties of the optimal strike $K^{*}$ which uniquely satisfies $\partial H / \partial K=0$ in Eq.(4.17). The optimal strike $K^{*}$ has a graphical interpretation(see Fig.(1)): $\left(K^{*}, C\left(x, K^{*}\right)\right)$ is the point at which a line through two points $(B, 0)$ and $\left(K^{*}, C\left(x, K^{*}\right)\right)$ is tangent to the function $C(x, K)$.

We analyze how the optimal strike $K^{*}$ is affected by the underlying price $S$ and its volatility $\sigma$ that are main factors for pricing the options. We assume the following assumption which usually holds true.

Assumption 4. Suppose that g defined as Eq.(4.19) is strictly increasing with respect to $S$ and $\sigma$ (some components of a $N$-dimensional vector $x$ ).

Remark 5. Assumption 4 usually holds true because it is expected that the absolute variation of $\frac{\partial C}{\partial K}$ with respect to $S$ and $\sigma$ respectively is smaller than that of $C$.

Then, we obtain the following property of $K^{*}$.

Proposition 2. Suppose Assumption 4 holds. Then $\frac{\partial K_{s}^{*}}{\partial S} \frac{\partial K_{s}^{*}}{\partial \sigma}>0$. 


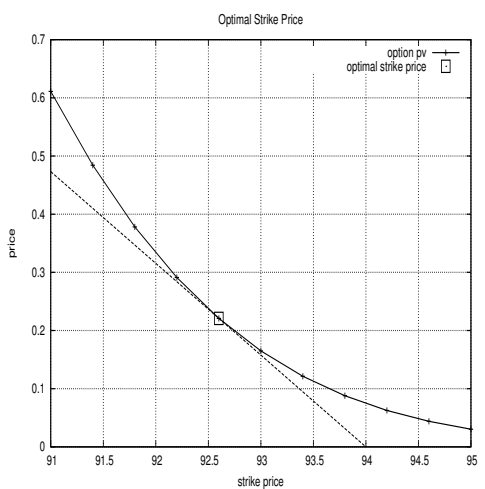

Figure 1: The optimal strike $(B=94)$

Proof. Let the price of a European call option be $C(\alpha, K)$ with $\alpha=S$ or $\sigma$ for short, where we ignore other market parameters and $K_{\alpha}^{*}$ be the strike price which gives the unique zero for the equation $g(\alpha, K)=0$. Then, we have $g(\cdot, 0)=S-B<0$. Let us consider the sign of $g\left(\beta, K_{\alpha}^{*}\right)$ for $\beta>\alpha$. Assumption 4 implies

$$
\begin{aligned}
g\left(\beta, K_{\alpha}^{*}\right) & =\frac{\partial C}{\partial K}\left(\beta, K_{\alpha}^{*}\right)\left(B-K_{\alpha}^{*}\right)+C\left(\beta, K_{\alpha}^{*}\right) \\
& >\frac{\partial C}{\partial K}\left(\alpha, K_{\alpha}^{*}\right)\left(B-K_{\alpha}^{*}\right)+C\left(\alpha, K_{\alpha}^{*}\right)=0 .
\end{aligned}
$$

Therefore, $K_{\beta}^{*}$ must be uniquely in the interval $\left(0, K_{\alpha}^{*}\right)$ by the continuity of $g$, which leads to the fact that $K_{\alpha}^{*}$ is strictly decreasing with respect to $\alpha$.

Remark 6. Proposition 2 shows that the integrand $\frac{\partial K_{s}^{*}}{\partial S} \frac{\partial K_{s}^{*}}{\partial \sigma}$ of the last term in the following approximation is positive:

$$
\begin{aligned}
\left\langle K^{*}\right\rangle_{t} \approx & \int_{0}^{t}\left(\frac{\partial K_{s}^{*}}{\partial S}\right)^{2} d\langle S\rangle_{s}+\int_{0}^{t}\left(\frac{\partial K_{s}^{*}}{\partial \sigma}\right)^{2} d\langle\sigma\rangle_{s} \\
& +\int_{0}^{t} \frac{\partial K_{s}^{*}}{\partial S} \frac{\partial K_{s}^{*}}{\partial \sigma} d\langle S, \sigma\rangle_{s} .
\end{aligned}
$$

Hence, roughly speaking, the quadratic variation of $K^{*}$ is positively correlated to the quadratic covariation of $S$ and $\sigma$.

\section{$5 \quad$ Numerical Examples}

In this section, we implement two types of Monte Carlo simulation tests of the dynamically rebalancing super-replication for cross-currency options: the purpose of the first simulation is to confirm that it can be used as an investment strategy on the correlation as stated in Remark 4, and the second is intended to demonstrate the effectiveness of our strategy in 
hedging through comparing a hedging performance of our strategy with those of other hedging strategies.

Consider a trading strategy where the dynamically rebalancing superreplication is applied against shorting an ATM cross-currency option. For example, let a currency exchange rate $S_{t}^{a}\left(S_{t}^{j}\right)$ represent a time- $t$ price of the unit amount of USD in terms of $\operatorname{AUD}(\mathrm{JPY})^{3}$. Consider a crosscurrency rate representing the price of the unit amount of AUD in terms of JPY. Then, the payoff of a call option on the cross-currency with strike $\operatorname{spot} \operatorname{ATM}\left(S_{0}^{j} / S_{0}^{a}\right)$ and maturity $T$ in terms of JPY is given by

$$
\left(\frac{S_{T}^{j}}{S_{T}^{a}}-\frac{S_{0}^{j}}{S_{0}^{a}}\right)_{+}=S_{T}^{j} \cdot \frac{1}{S_{0}^{a}}\left(\frac{S_{0}^{a}}{S_{T}^{a}}-\frac{S_{0}^{j}}{S_{T}^{j}}\right)_{+}
$$

In the following simulations, we normalize the processes of the exchange rates so that $S_{0}^{a}=S_{0}^{j}=1$.

\subsection{Investment on Correlation}

In order to focus on a correlation investment, we adopt a simple model that is a correlated log-normal model with a constant correlation as in the following assumption.

Assumption 5. The processes of the exchange rates $S_{t}^{j}$ and $S_{t}^{a}$ are assumed to be correlated log-normal with constant volatilities and a constant correlation:

$$
\begin{aligned}
d S_{t}^{j} & =\sigma^{j} S_{t}^{j} d W_{t}^{j} \\
d S_{t}^{a} & =\sigma^{a} S_{t}^{a} d W_{t}^{a}, \\
\left\langle W^{j}, W^{a}\right\rangle_{t} & =\rho t,
\end{aligned}
$$

where $W_{t}^{j}$ and $W_{t}^{a}$ are 1 dimensional Brownian motions and $\sigma^{j}, \sigma^{a}$ and $\rho$ are constant.

Our simulation settings are listed in Table 1, where we have two values of the correlation in order to see how the performance of the strategy is affected by the correlation. We sell 100.0 units of a cross-currency option at $14.14 \%$ implied volatility, which corresponds to $\rho=-0.25$ and rabalance static portfolios every five days.

Table 1: Settings of the simulation

\begin{tabular}{cccccc}
\hline$T$ & $S_{0}^{j}$ & $S_{0}^{a}$ & $\sigma^{j}$ & $\sigma^{a}$ & $\rho$ \\
\hline 30 (days) & 1.0 & 1.0 & $10.0(\%)$ & $10.0(\%)$ & $0.0 /-0.5$ \\
\hline
\end{tabular}

Fig.2 shows the result of the simulations in terms of JPY; it shows histograms of the performance corresponding to the correlation values. It is found that the higher is the correlation, the more profit is obtained, which is consistent with Remark 4.

${ }^{3}$ AUD and JPY stand for Australian dollar and Japanese yen, respectively. 


\subsection{Effectiveness as a Hedging Strategy}

This subsection considers hedging as an application of the dynamically rebalancing super-replication. Especially, hedging a short position by the strategy seems attractive to risk-averse investors. The strategy has two distinctive features: one is to avoid substantial losses and another is to prevent the worst-case scenario which would often occur if rebalancing would not be carried out.

In order to demonstrate those, a hedging performance of the strategy is compared with those of two other hedging strategies; Black-Scholes dynamic hedging and the static super-replication of Chung and Wang [2], which is a static position introduced in Lemma 2. We implement a simulation test where paths are generated by a realistic model, where the volatilities of both exchange rates are stochastic. The following model is used for generating paths of the simulation.

Assumption 6. The processes of the exchange rates $S_{t}^{j}$ and $S_{t}^{a}$ are assumed to follow the model:

$$
\begin{aligned}
d S_{t}^{j} & =S_{t}^{j} \sigma_{t}^{j} d W_{t}^{j} \\
d S_{t}^{a} & =S_{t}^{a} \sigma_{t}^{a} d W_{t}^{a},
\end{aligned}
$$

where $\zeta_{t}^{j}:=\log \sigma_{t}^{j}$ and $\zeta_{t}^{a}:=\log \sigma_{t}^{a}$ follow

$$
\begin{aligned}
d \zeta_{t}^{j} & =\xi^{j}\left(\eta^{j}-\zeta_{t}^{j}\right) d t+\theta^{j} d Z_{t}^{j}, \\
d \zeta_{t}^{a} & =\xi^{a}\left(\eta^{a}-\zeta_{t}^{a}\right) d t+\theta^{a} d Z_{t}^{a} .
\end{aligned}
$$

$\left(\xi^{j}, \eta^{j}, \theta^{j}\right)$ and $\left(\xi^{a}, \eta^{a}, \theta^{a}\right)$ are constant and $W^{j}, W^{a}, Z^{j}$ and $Z^{a}$ are 1 dimensional Brownian motions with

$$
d\left\langle W^{j}, W^{a}\right\rangle=\rho, d\left\langle W^{j}, Z^{j}\right\rangle=\rho^{j}, d\left\langle W^{a}, Z^{a}\right\rangle=\rho^{a},
$$

where the other correlations are zero.

Our simulation settings are listed in Table 2. We sell 100.0 units of a cross-currency option at $20.18 \%$ implied volatility that is computed by $\sigma_{i v}^{j}=14.0 \%, \sigma_{i v}^{a}=16.0 \%$ and $\rho=0.1$. Then, we rebalance static portfolios every five days while rebalancing the delta every day for BlackScholes hedging, where the delta is evaluated with $20.18 \%$ cross-currency rate volatility. The implied volatilities are set to be flat with $14.0 \%$ and $16.0 \%$.

Table 2: Settings of the simulation

\begin{tabular}{cccccc}
\hline$T$ & $S_{0}^{j}$ & $S_{0}^{a}$ & $\rho$ & $\sigma_{i v}^{j}$ & $\sigma_{i v}^{a}$ \\
\hline 30 (days) & 1.0 & 1.0 & 0.1 & $14.0(\%)$ & $16.0(\%)$ \\
\hline & $\sigma_{0}^{*}$ & $\xi^{*}$ & $\eta^{*}$ & $\theta^{*}$ & $\rho^{*}$ \\
\hline$j$ & $9.50(\%)$ & 347.22 & -2.75 & 23.57 & -0.0011 \\
$r$ & $11.93(\%)$ & 311.08 & -2.7 & 23.3 & 0.0015 \\
\hline
\end{tabular}


Table 3: Statistics of Hedging Errors(yen)

\begin{tabular}{c|cccccccc}
\hline Strategy & Mean & Std Err & Mode & Min & $1 \%$ & $5 \%$ & $10 \%$ & $25 \%$ \\
\hline B.S. & 0.03 & 1.22 & 0.43 & -23.05 & -4.59 & -2.03 & -1.22 & -0.35 \\
C\&W & 0.00 & 1.77 & -1.12 & -1.12 & -1.12 & -1.12 & -1.12 & -1.12 \\
out strategy & -0.05 & 1.39 & -0.98 & -1.12 & -1.11 & -1.05 & -1.00 & -0.84 \\
\hline
\end{tabular}

Fig. 3 shows histograms of the performances of the strategies and Table 3 shows their statistics, where $\mathrm{C} \& \mathrm{~W}$ stands for Chung and Wang [2]. First, while there are substantial losses(over 2.0yen) in the results of B.S., the maximum loss is 1.12 yen in our strategy, which means that out strategy can avoid substantial losses. Second, it is found from Fig.3 that almost half scenarios of the strategy of Chung and Wang [2] are the worst, where the maximum loss is 1.12 yen. On the other hand, our strategy mostly avoids the worst case and achieve improvements in VaR over the Chung and Wang [2](see Table 3). Consequently, it is confirmed that our strategy can avoid substantial losses and mostly prevent the worst case scenario of Chung and Wang [2].

\section{Concluding Remarks}

We introduced a trading strategy that dynamically rebalances superreplicating portfolios; this strategy is attractive for both investment and hedging. Then, without assuming any models under the continuous processes of the underlying variables, we derived the Doob-Meyer decomposition for the value process of this strategy to obtain the general properties: specifically, we found that the performance of the strategy is characterized by the the increasing part of the decomposition. Also, our general framework was successfully applied to cross-currency and one-touch options, which provides more concrete implications in practice. Moreover, numerical examples for cross-currency options confirmed the property shown in the previous sections, and demonstrated our strategy is useful for hedging under stochastic volatility environment.

Finally, our next research topic will be to analyze properties of the dynamics of the optimal parameter $K^{*}$ and to evaluate the expectation of the increasing part of the super-martingale process in order to calculate a price of the option based on the strategy. Also an extension of our result to discontinuous processes of the underlying variables is an interesting theme.

\section{A Analytical Results for Exchange Op- tions under Black-Scholes Model}

In this section, we derive analytical results under the Black-Scholes model of exchange options. For simplicity, we express the price of asset 1 and as- 
set 2 with $X_{t}$ and $Y_{t}$ respectively instead of $X_{t}^{(i)}$. We assume the following assumption.

Assumption 7. The processes of both asset 1 and asset 2 are assumed to be log-normal with constant volatility:

$$
\begin{aligned}
d X_{t} & =\sigma_{X} X_{t} d W_{t}^{X} \\
d Y_{t} & =\sigma_{Y} Y_{t} d W_{t}^{Y},
\end{aligned}
$$

where $W_{t}^{X}, W_{t}^{Y}$ are 1 dimensional Brownian motions under the risk neutral measure $\mathbb{Q}$, and $\sigma_{X}$ and $\sigma_{Y}$ are constant.

In addition, we define some notations:

$$
\begin{array}{r}
N(x)=\frac{1}{\sqrt{2 \pi}} \int_{-\infty}^{x} e^{-\frac{y^{2}}{2}} d y \\
d_{X}^{ \pm}=\frac{\log \frac{x}{K} \pm \sigma_{X}^{2} \tau}{\sigma_{X} \sqrt{\tau}} \\
d_{Y}^{ \pm}=\frac{\log \frac{y}{K} \pm \sigma_{Y}^{2} \tau}{\sigma_{Y} \sqrt{\tau}}
\end{array}
$$

where $\tau=T-t$.

First, we derive the optimal strike price and the value of optimal portfolio for the super-replication and the sub-replication respectively.

Proposition 3. The optimal strike of the super-replication is:

$$
K^{G}(t, x, y)=x^{\frac{\sigma_{Y}}{\sigma_{X}+\sigma_{Y}}} y^{\frac{\sigma_{X}}{\sigma_{X}+\sigma_{Y}}} e^{-\frac{1}{2} \sigma_{X} \sigma_{Y} \tau},
$$

and the upper bound of an exchange option is:

$$
C_{B S}\left(t, x, y,\left|\sigma_{X}+\sigma_{Y}\right|\right) .
$$

Proof. By Eq.(4.8), $K^{G}(t, x, y)$ must satisfy $\frac{\partial G}{\partial K}\left(t, x, y, K_{t}^{G}(t, x, y)\right)=0$. So, $d_{X}^{-}=-d_{Y}^{-}$. Then, we get (A.6).

The value of super-replicating portfolio is:

$$
\begin{aligned}
& C^{X}(t, x, K)+P^{Y}(t, y, K) \\
= & x N\left(d_{X}^{+}\right)-K N\left(d_{X}^{-}\right)+K N\left(-d_{Y}^{-}\right)-y N\left(-d_{Y}^{+}\right) \\
= & x N\left(d_{X}^{+}\right)-y N\left(-d_{Y}^{+}\right) \\
= & x N\left(d_{1}\right)-y N\left(d_{2}\right) \\
= & C_{B S}\left(t, x, y, \sigma_{X}+\sigma_{Y}\right),
\end{aligned}
$$

where $K=K^{G}(t, x, y), d_{1}=\frac{\log \frac{x}{y}+\frac{1}{2}\left(\sigma_{X}+\sigma_{Y}\right)^{2} \tau}{\left(\sigma_{X}+\sigma_{Y}\right) \sqrt{\tau}}, d_{2}=\frac{\log \frac{x}{y}-\frac{1}{2}\left(\sigma_{X}+\sigma_{Y}\right)^{2} \tau}{\left(\sigma_{X}+\sigma_{Y}\right) \sqrt{\tau}}$.

Proposition 4. Assume $\sigma_{X}<\sigma_{Y}$. The optimal strike of the sub-replication is:

$$
K^{L}(t, x, y)=x^{\frac{-\sigma_{Y}}{\sigma_{X}-\sigma_{Y}}} y^{\frac{\sigma_{X}}{\sigma_{X}-\sigma_{Y}}} e^{\frac{1}{2} \sigma_{X} \sigma_{Y} \tau}
$$

and the lower bound of an exchange option is:

$$
C_{B S}\left(t, x, y,\left|\sigma_{X}-\sigma_{Y}\right|\right) \text {. }
$$


Proof. Put $d_{X}=d_{X}^{+}, d_{Y}=d_{Y}^{+}$, we have

$$
\begin{aligned}
\frac{\partial^{2} C^{X}}{\partial K^{2}}(t, K) & =\frac{1}{\sqrt{2 \pi}} \frac{x}{K^{2} \sigma_{X} \sqrt{\tau}} e^{-\frac{1}{2} d_{X}^{2}} \\
\frac{\partial^{2} C^{Y}}{\partial K^{2}}(t, K) & =\frac{1}{\sqrt{2 \pi}} \frac{y}{K^{2} \sigma_{Y} \sqrt{\tau}} e^{-\frac{1}{2} d_{Y}^{2}} .
\end{aligned}
$$

In order to investigate the sign of $\frac{\partial L^{C}}{\partial K}$ and $\frac{\partial L^{P}}{\partial K}$, we define the ratio $\psi(K)$ :

$$
\begin{aligned}
\psi(K) & =\frac{\frac{\partial^{2} C^{X}}{\partial K^{2}}(K)}{\frac{\partial^{2} C^{Y}}{\partial K^{2}}(K)} \\
& =\frac{x}{y} \frac{\sigma_{Y}}{\sigma_{X}} e^{-\frac{1}{2}\left(d_{X}^{2}-d_{Y}^{2}\right)} .
\end{aligned}
$$

There are at least two roots of the equation $\psi(K)=1$, because $\int_{0}^{+\infty} \frac{\partial^{2} C^{X}}{\partial K^{2}}(K) d K=$ $\int_{0}^{+\infty} \frac{\partial^{2} C^{Y}}{\partial K^{2}}(K) d K=1$ and $\lim _{K \rightarrow 0} \psi(K)=\lim _{K \rightarrow+\infty} \psi(K)=0$ by the assumption $\sigma_{X}<\sigma_{Y}$. Consider the sign of $\frac{\partial \psi}{\partial K}$ :

$$
\begin{aligned}
\frac{\partial \psi}{\partial K}(K) & =\frac{2 \psi(K)}{K}\left(\frac{\log \frac{x}{K}+\frac{1}{2} \sigma_{X}^{2} \tau}{\sigma_{X}^{2} \tau}-\frac{\log \frac{y}{K}+\frac{1}{2} \sigma_{Y}^{2} \tau}{\sigma_{Y}^{2} \tau}\right) \\
& =\frac{2 \psi(K)}{K \tau}\left(\left(\frac{1}{\sigma_{Y}^{2}}-\frac{1}{\sigma_{X}^{2}}\right) \log K+\frac{1}{\sigma_{X}^{2}} \log x-\frac{1}{\sigma_{Y}^{2}} \log y\right)
\end{aligned}
$$

Then, the number of the roots of the equation $\psi(K)=1$ is exactly two.

The fact that $\frac{\partial^{2} L^{C}}{\partial K^{2}}>0$ is equivalent to $\psi(K)>1$ and $\lim _{K \rightarrow 0} \frac{\partial L^{C}}{\partial K}=$ $\lim _{K \rightarrow+\infty} \frac{\partial L^{C}}{\partial K}=0$ implies that the equation $\frac{\partial L^{C}}{\partial K}=0$ has an unique solution. By the same reason, the equation $\frac{\partial L^{P}}{\partial K}=0$ has an unique solution.

The definition of $L^{C}(t, x, y, K)$ and $L^{P}(t, x, y, K)$ shows that

$$
\begin{aligned}
& \lim _{K \rightarrow 0} L^{C}(t, x, y, K)=x-y \\
& \lim _{K \rightarrow 0} L^{P}(t, x, y, K)=0,
\end{aligned}
$$

and

$$
\begin{aligned}
\lim _{K \rightarrow+\infty} L^{C}(t, x, y, K) & =0 \\
\lim _{K \rightarrow+\infty} L^{P}(t, x, y, K) & =x-y .
\end{aligned}
$$

As a result, we find that $L^{C}(t, x, y, K)$ has only one local minimum and $L^{P}(t, x, y, K)$ has only one local maximum.

In order to compare a maximum of $L^{P}(t, x, y, K)$ with that of $L^{C}(t, x, y, K)$, we calculate the maximum of $L^{P}(t, x, y, K)$. Let $K_{*}$ be the solution of the equation $\frac{\partial L^{P}}{\partial K}(t, x, y, K)=0$. By $\frac{\partial P^{X}}{\partial K}(t, x, y, K)=\frac{\partial P^{Y}}{\partial K}(t, x, y, K)$, we can derive $d_{X}^{-}=d_{Y}^{-}$and then,

$$
K_{*}=x^{\frac{-\sigma_{Y}}{\sigma_{X}-\sigma_{Y}}} y^{\frac{\sigma_{X}}{\sigma_{X}-\sigma_{Y}}} e^{\frac{1}{2} \sigma_{X} \sigma_{Y} \tau} .
$$


The maximum of $L^{P}(t, x, y, K)$ is:

$$
\begin{aligned}
& -P^{X}(t, x, K)+P^{Y}(t, y, K) \\
= & -\left(K N\left(-d_{X}^{-}\right)-x N\left(-d_{X}^{+}\right)\right)+\left(K N\left(-d_{Y}^{-}\right)-y N\left(-d_{Y}^{+}\right)\right) \\
= & x N\left(-d_{X}^{+}\right)-y N\left(-d_{Y}^{+}\right) \\
= & x N\left(d_{1}\right)-y N\left(d_{2}\right) \\
= & C_{B S}\left(x, y,\left|\sigma_{X}-\sigma_{Y}\right|\right),
\end{aligned}
$$

where $K=K_{*}, d_{1}=\frac{\log \frac{x}{y}+\frac{1}{2}\left(\sigma_{X}-\sigma_{Y}\right)^{2} \tau}{\left|\sigma_{X}-\sigma_{Y}\right| \sqrt{\tau}}$ and $d_{2}=\frac{\log \frac{x}{y}-\frac{1}{2}\left(\sigma_{X}-\sigma_{Y}\right)^{2} \tau}{\left|\sigma_{X}-\sigma_{Y}\right| \sqrt{\tau}}$. This maximum is bounded below by $(x-y)_{+}$.

Finally, we conclude that the optimal strike is:

$$
K^{L}(t, x, y)=K_{*},
$$

and the lower bound of an exchange option is:

$$
\sup _{K>0} \max \left\{L^{C}(t, x, y, K), L^{P}(t, x, y, K)\right\}=L^{P}\left(t, x, y, K^{L}(x, y)\right) .
$$

By the proof of Proposition 4, we have the following proposition, which means that Assumption 3 holds true for sub-replication under a certain condition.

Proposition 5. Assume $\sigma_{X}<\sigma_{Y}$, then the optimal strike price of sub replicating portfolio is determined uniquely. $L(t, x, y, K)$ can be differentiated with respect to $x, y$ and $K$ in the neighborhood of $K=K^{L}(t, x, y)$ and $K^{L}(t, x, y)$ can be differentiated with respect to $x, y$. Moreover, we have

$$
\begin{aligned}
\frac{\partial L}{\partial K}\left(t, x, y, K^{L}(t, x, y)\right) & =0 \\
\frac{\partial^{2} L}{\partial K^{2}}\left(t, x, y, K^{L}(t, x, y)\right) & >0 .
\end{aligned}
$$

We have the Doob-Meyer decomposition explicitly.

Corollary 1. Assume Assumption 7 and

$$
\left\langle W^{X}, W^{Y}\right\rangle_{t}=\rho t
$$

where $\rho \in[-1,1]$ is constant. Let $\psi$ be the probability density function for the standard normal distribution : $\psi(x)=\frac{1}{\sqrt{2 \pi}} e^{-\frac{x^{2}}{2}}$.

Then, in case of the super-replication,

$$
\begin{gathered}
A_{t}^{*}=\int_{0}^{t} \frac{1+\rho}{\sqrt{T-s}}\left(\frac{X_{s}}{\sigma_{X}} \psi\left(d_{X}^{G}\right)+\frac{Y_{s}}{\sigma_{Y}} \psi\left(d_{Y}^{G}\right)\right)\left(\frac{\sigma_{X} \sigma_{Y}}{\sigma_{X}+\sigma_{Y}}\right)^{2} d s \\
M_{t}^{*}=\int_{0}^{t}\left(\frac{\partial C^{X}}{\partial x}\left(s, X_{s}, K_{s}^{G}\right) d X_{s}+\frac{\partial P^{Y}}{\partial y}\left(s, Y_{s}, K_{s}^{G}\right) d Y_{s}\right)
\end{gathered}
$$


where

$$
\begin{aligned}
& d_{X}^{G}:=\frac{\log \frac{X_{s}}{Y_{s}}+\frac{1}{2}\left(\sigma_{X}+\sigma_{Y}\right)^{2}(T-s)}{\left(\sigma_{X}+\sigma_{Y}\right) \sqrt{T-s}}, \\
& d_{Y}^{G}:=\frac{\log \frac{X_{s}}{Y_{s}}-\frac{1}{2}\left(\sigma_{X}+\sigma_{Y}\right)^{2}(T-s)}{\left(\sigma_{X}+\sigma_{Y}\right) \sqrt{T-s}}, \\
& K_{s}^{G}:=X_{s}^{\frac{\sigma_{Y}}{\sigma_{X}+\sigma_{Y}}} Y_{s}^{\frac{\sigma_{X}}{\sigma_{X}+\sigma_{Y}}} e^{-\frac{1}{2} \sigma_{X} \sigma_{Y}(T-s)} .
\end{aligned}
$$

In case of the sub-replication with $\sigma_{X}<\sigma_{Y}$,

$$
\begin{gathered}
A_{t}^{*}=\int_{0}^{t} \frac{1-\rho}{\sqrt{T-s}}\left(\frac{X_{s}}{\sigma_{X}} \psi\left(d_{X}^{L}\right)-\frac{Y_{s}}{\sigma_{Y}} \psi\left(d_{Y}^{L}\right)\right)\left(\frac{\sigma_{X} \sigma_{Y}}{\sigma_{X}-\sigma_{Y}}\right)^{2} d s \\
M_{t}^{*}=\int_{0}^{t}\left(\frac{\partial P^{X}}{\partial x}\left(s, X_{s}, K_{s}^{L}\right) d X_{s}-\frac{\partial P^{Y}}{\partial y}\left(s, Y_{s}, K_{s}^{L}\right) d Y_{s}\right)
\end{gathered}
$$

where

$$
\begin{aligned}
d_{X}^{L} & :=\frac{\log \frac{X_{s}}{Y_{s}}+\frac{1}{2}\left(\sigma_{X}-\sigma_{Y}\right)^{2}(T-s)}{\left(\sigma_{X}-\sigma_{Y}\right) \sqrt{T-s}} \\
d_{Y}^{L} & :=\frac{\log \frac{X_{s}}{Y_{s}}-\frac{1}{2}\left(\sigma_{X}-\sigma_{Y}\right)^{2}(T-s)}{\left(\sigma_{X}-\sigma_{Y}\right) \sqrt{T-s}} \\
K_{s}^{L} & :=X_{s}^{\frac{-\sigma_{Y}}{\sigma_{X}-\sigma_{Y}}} Y_{s}^{\frac{\sigma_{X}}{\sigma_{X}-\sigma_{Y}}} e^{\frac{1}{2} \sigma_{X} \sigma_{Y}(T-s)}
\end{aligned}
$$

Proof. Combine Proposition 3, 4 and Theorem 1.

\section{Acknowledgment}

The authors are grateful to Mr. Toshifumi Ikemori(Mizuho-DL Financial Technology Co., Ltd.), Mr. Tsuyoshi Hase(Mizuho-DL Financial Technology Co., Ltd.), Mr. Shuji Munekuni(Mizuho-DL Financial Technology Co., Ltd.), Mr. Seiichi Tanaka(Mizuho Corporate Bank, Ltd.), and Mr. Takehiko Ariga(Mizuho Corporate Bank, Ltd.) for their constant support in implementing this research. The views expressed in the paper are those of the authors and do not necessarily reflect those of Mizuho-DL Financial Technology Co., Ltd.

\section{References}

[1] H. Brown, D. Hobson, and L. C. G. Rogers. "Robust Hedging of Barrier Options". Mathematical Finance, 11(3):285 - 314, 2001.

[2] S-L. Chung and Y-H. Wang. "Bounds and prices of currency cross-rate options". Journal of Banking \& Finance, 32(5):631-642, May 2008.

[3] B. Dupire. "The Return(s) Of Super-Replication". 2010. Presentation given at the ICBI Global Derivatives conference, Paris, 19th May 2010. 
[4] N. El Karoui and M. Quenez. "Dynamic Programming and Pricing of Contingent Claims in an Incomplete Market". SIAM Journal on Control and Optimization, 33:29-66, Jan. 1995.

[5] W. Margrabe. "The Value of an Option to Exchange One Asset for Another". Journal of Finance, 33:177-186, 1978.

[6] A. Neuberger and S. Hodges. "Rational Bounds on the Prices of Exotic Options". http://papers.ssrn.com/abstract=239711, 2000.

[7] Y. Tsuzuki. "Super-Hedging with Young's Inequality". http://papers.ssrn.com/abstract=1755274, 2011. 

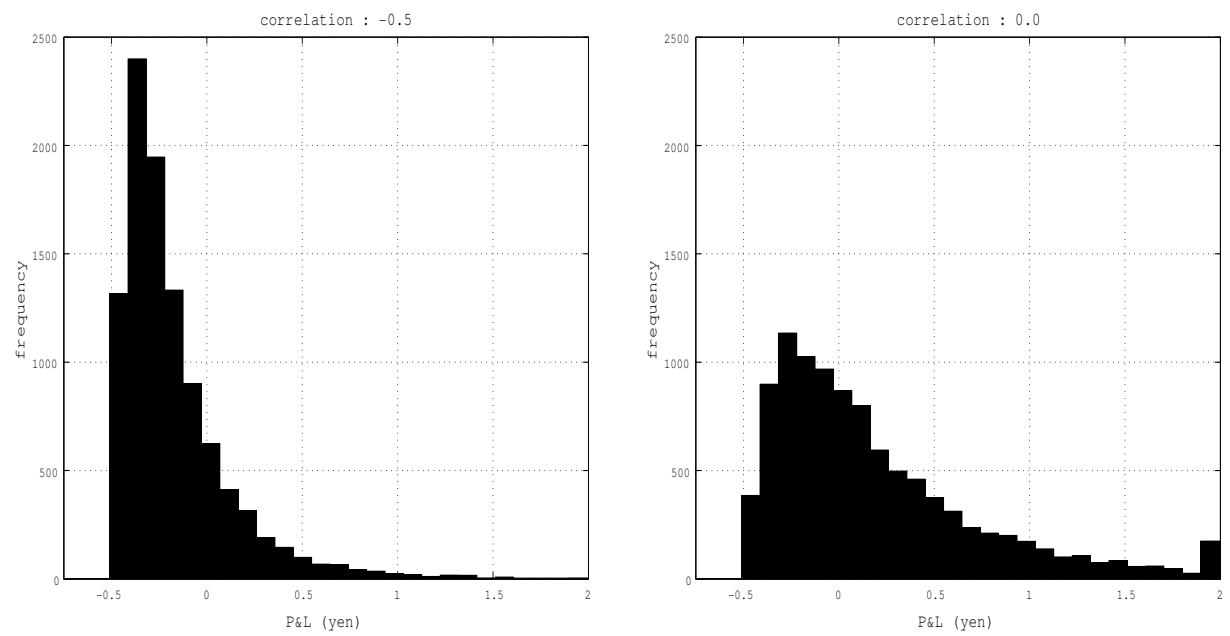

Figure 2: Comparison of Correlation
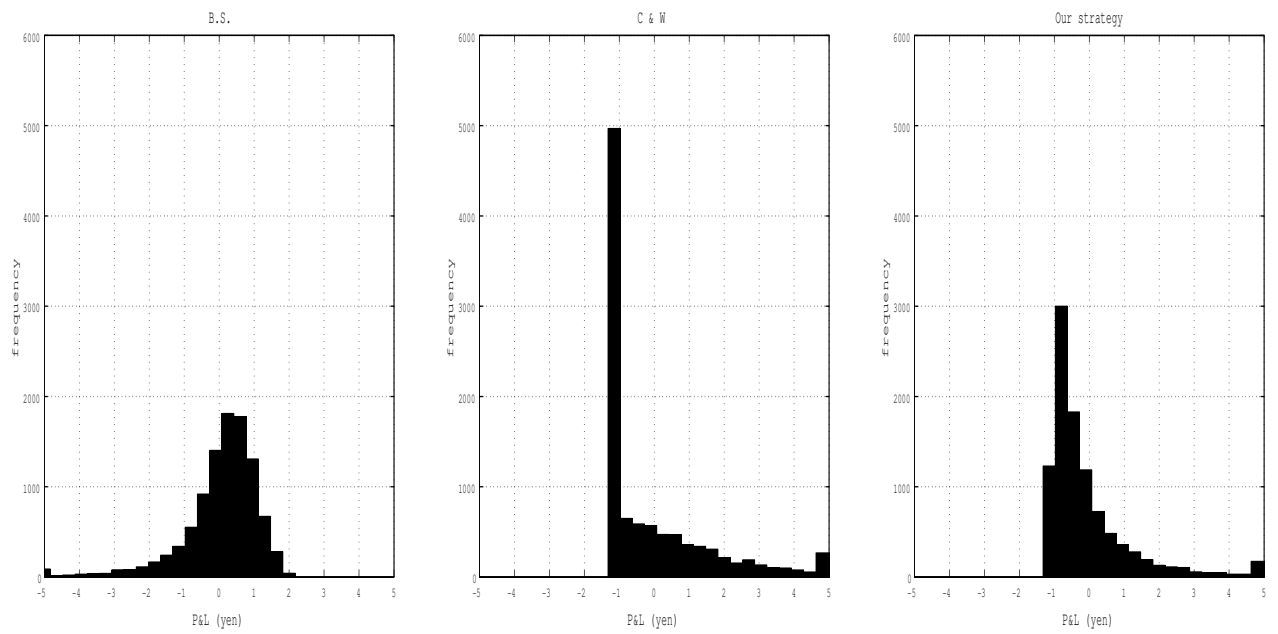

Figure 3: Comparison of Hedging Schems 